\title{
Torsional Alfvén waves in small scale current threads of the solar corona
}

\author{
P. Copil ${ }^{1}$, Y. Voitenko ${ }^{2}$, and M. Goossens ${ }^{1}$ \\ 1 Centre for Plasma Astrophysics, K. U. Leuven, Celestijnenelaan 200 B, 3001 Heverlee, Belgium \\ e-mail: copil@wis . kuleuven . be \\ 2 Space Physics Division, Belgian Institute for Space Aeronomy, Ringlaan-3-Avenue Circulaire, 1180 Brussels, Belgium
}

Received 29 June 2009 / Accepted 5 November 2009

\begin{abstract}
Context. The magnetic field structuring in the solar corona occurs on large scales (loops and funnels), but also on small scales. For instance, coronal loops are made up of thin strands with different densities and magnetic fields across the loop.

Aims. We consider a thin current thread and model it as a magnetic flux tube with twisted magnetic field inside the tube and straight field outside. We prove the existence of trapped Alfvén modes in twisted magnetic flux tubes (current threads) and we calculate the wave profile in the radial direction for two different magnetic twist models.

Methods. We used the Hall MHD equations that we linearized in order to derive and solve the eigenmode equation for the torsional Alfvén waves.

Results. We show that the trapped Alfvén eigenmodes do exist and are localized in thin current threads where the magnetic field is twisted. The wave spectrum is discrete in phase velocity, and the number of modes is finite and depends on the amount of the magnetic field twist. The phase speeds of the modes are between the minimum of the Alfvén speed in the interior and the exterior Alfén speed. Conclusions. Torsional Alfvén waves can be guided by thin twisted magnetic flux-tubes (current threads) in the solar corona. We suggest that the current threads guiding torsional Alfvén waves, are subject to enhanced plasma heating due to wave dissipation.
\end{abstract}

Key words. Sun: corona - magnetic fields - magnetohydrodynamics (MHD) - waves

\section{Introduction}

The Alfvén wave is one of the magnetohydrodynamic (MHD) waves propagating in magnetized plasmas. The MHD waves have been intensively studied by solar physicists, who predicted their existence in the solar atmosphere well before they had actually been observed. Theoretical modelling of MHD waves in structured magnetised plasma was developed more than twenty years ago by Spruit $(1981,1982)$, Roberts \& Webb (1979), Edwin \& Roberts (1983), and others. After the launching of space-based telescopes SOHO and TRACE, a huge amount of data from the solar atmosphere became available and the predictions could finally be tested. The observations confirmed that the fast kink (Aschwanden et al. 1999; Nakariakov et al. 1999; Wang \& Solanki 2004), sausage (Nakariakov et al. 2003; Aschwanden et al. 2004), and slow magnetoacoustic (De Moortel et al. 2002; Wang et al. 2003) waves are supported by magnetic plasma structures of the solar atmosphere.

Torsional Alfvén waves are more difficult to observe because, due to their near incompressibility, they do not produce density variations hence no variations in the observed emission intensity. However, it is still possible to detect them using spectroscopic measurements. The effects of torsional waves are line broadening for spatially unresolved velocities and line shifting for spatially resolved velocities. These are consequences of the Doppler shifts in the emitted radiation.

Observations made in coronal holes above the limb show that the line widths first increase with altitude up to 1.2 solar radii, exhibit a constant plateau up to 1.5 solar radii, and then start to decrease (Banerjee et al. 1998). At the equator, Harrison et al. (2002) also observed a decrease in line width above $50000 \mathrm{~km}$, with the width narrower than above coronal holes. The interpretation is that this behaviour is caused by Alfvén waves, with the increase in line width caused by wave flux conservation and decrease by wave damping. Very recent observations performed by Dolla \& Solomon (2008) show the same behaviour of the spectral lines. However, they suggest that line narrowing is not a consequence of wave damping, but is an instrumental effect caused by stray light.

Zaqarashvili (2003) suggests that the torsional Alfvén waves can be observed as temporal and spatial variations in spectral emission along the coronal loops. A standing wave has nodes and antinodes, so by measuring the distance between two nodes or two antinodes, the wavelength $\lambda$ of the standing wave can be estimated. After the wave period, $T$ is measured from observations, the Alfvén speed in the corona can be computed using the relation $v_{\mathrm{A}}=\lambda / T$. By using early observations by Egan \& Schneeberger (1979) of periodic Doppler width fluctuations in Fe XIV spectra above an active region, Zaqarashvili calculated the Alfvén speed and the amplitude of the torsional Alfvén wave in a coronal loop.

In this paper, we study analytically the torsional Alfvén eigenmodes in the coronal loops, particularly in thin current threads composing the loops. Coronal loops were observed for the first time in the 70s with the Skylab mission (Vaiana et al. 1973). The images revealed that the corona is highly stratified in density and magnetic field, and coronal plasma is concentrated mostly in loops. More recent pictures taken with TRACE show 
that coronal loops are also structured, consisting of fine strands, as long as the loop, but about $2000 \mathrm{~km}$ in width (Aschwanden \& Nightingale 2005). More recent Hinode observations also suggest that coronal loops consist of multiple threads (Ofman \& Wang 2008). The threads are outlined by chromospheric material spreading along them. Ofman \& Wang (2008) looked at transverse loop oscillations, which were interpreted to be the fast magnetoacoustic waves. These waves have different properties in different threads, such that they are standing in some threads and in others propagating.

The loops are formed in a strong magnetic field, which varies in the range 10-100 Gauss (Aschwanden et al. 2001). There is also evidence that the magnetic field inside the loops is twisted. Leka et al. (1996) investigated five different loops and analysed their morphology and footpoint motion. They suggest that the magnetic field of the loops was already twisted before it emerged in the photosphere. Analysis of the sunspot motion led Ishii et al. (1998) to conclude that the twisted flux tubes are formed in the convection zone before they emerge above the photosphere. These authors also propose a schematic model for the magnetic field in the solar atmosphere, where they point out that the geometry of the magnetic field in the corona is very complex. The field that they deduced from their data appeared twisted, coiling around the trunk of a magnetic flux tube.

Modelling of MHD waves in twisted magnetic flux-tubes was done by Erdélyi \& Carter (2006). They consider a threelayer cylinder in which the magnetic field is straight in the interior, twisted in a finite layer, and then again straight in the exterior. The authors find that the twist introduces the so-called hybrid surface-body MHD modes. The hybrid modes are bodylike in the twisted part and evanescent in the part where the magnetic field is straight. Ofman (2009) constructed a 3D model of a coronal loop, made up of four threads. By perturbing the loop with a velocity pulse at the footpoints, the loop starts oscillating in a fundamental mode that resembles the kink mode of a single threaded loop. If the four-threaded loop is twisted, the induced oscillation is complex with all three components of the velocity generated in the loop. In the twisted case, the oscillation is no longer described by the fundamental kink mode.

The present work aims to investigate the trapped Alfvén waves in thin, twisted magnetic flux-tubes (current threads) with homogeneous density, but with an inhomogeneous magnetic field. Since there is a lot of evidence of twisted magnetic fields in the solar corona, we assume that the inhomogeneity is introduced by the non-uniform azimuthal component of the magnetic field.

The paper is structured as follows. In the second section we present the model for the coronal loop and the equations used to mathematically describe the torsional Alfvén waves. In the third section we present and explain the results and the last section contains conclusions and plans for future work.

\section{Current thread model and governing equations}

We model the coronal current thread as a straight cylinder with the same homogeneous density inside and outside the cylinder. The magnetic field is varies, such that it is straight at the axis $(r=0)$, then twists up to a radius $R$, and is again straight and constant in the exterior of the tube. Inside the tube, the magnetic field, denoted by $\boldsymbol{B}_{0}$, has an azimuthal component, $B_{0 \varphi}(r)$, and a field-aligned component, $B_{0 z}(r)$. For $r>R, B_{\varphi}=0$ and $B_{0 z}$ is constant. We consider very low values of $R$, of the order of several tens of meters. Such thin threads can result from the filamentation of larger-scale coronal currents or can be generated by

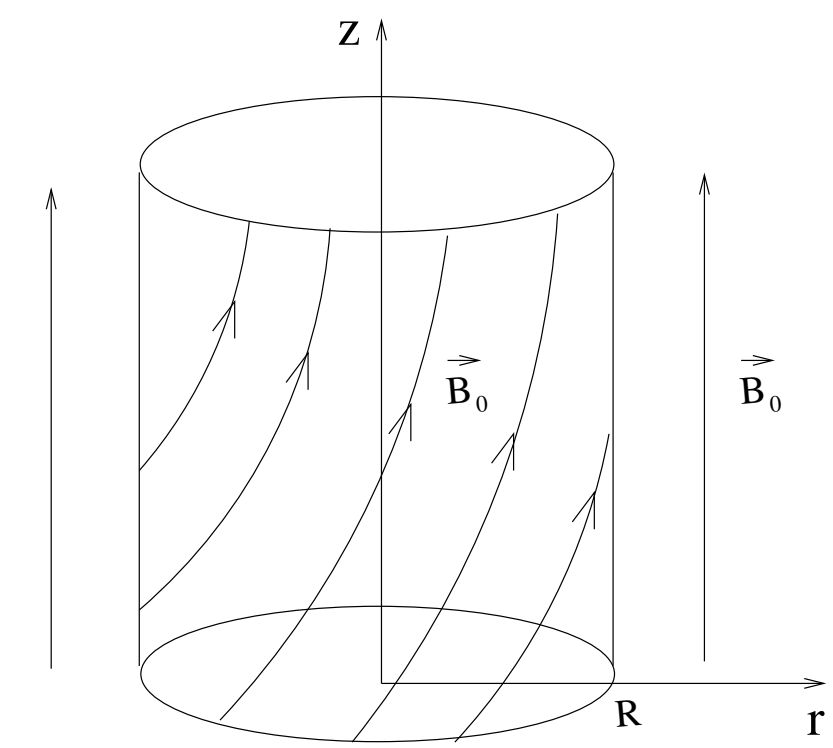

Fig. 1. Model of a current thread. The plasma density is constant everywhere, but the magnetic field is changing, such that it is straight at $r=0$, then it twists, and then it is straight again at $r \geq R$.

the small-scale photospheric vortices with the consequent pinching of the currents at coronal heights. The equilibrium is plotted in Fig. 1.

Eversince they were discovered theoretically by H. Alfvén in 1942 , and confirmed experimentally by S. Lundquist in 1949, the shear Alfvén waves have become a heavily studied wave mode in astrophysical and laboratory plasmas. These are low-frequency waves in plasmas embedded in the background magnetic field, with the main wave components the co-aligned plasma motions and magnetic field perturbations perpendicular to the background magnetic field.

In the ideal MHD model for a plasma embedded in a straight magnetic field, the perturbed velocity, $v_{\perp}$, and the magnetic field perturbation, $b_{\perp}$, are the only componets of the shear Alfvén wave. In a homogeneous plasma, the shear wave can have any profile in the direction perpendicular to the magnetic field, while it has a sinusoidal shape along the field, $\sim \sin \left(k_{z} z-\omega t\right)$, propagating with the Alfvén speed, $\omega / k_{z}=v_{\mathrm{A}}\left(v_{\mathrm{A}}=B_{0} / \sqrt{4 \pi \rho_{0}}, B_{0}\right.$ is the background magnetic field, and $\rho_{0}$ is the plasma density). If the plasma is inhomogeneous in the cross-field plane, there is a continuous spectrum of the waves with the same wavenumber $k_{z}$ but with different frequencies. On each magnetic surface $r=r_{0}$, where the Alfvén velocity $v_{\mathrm{A}}\left(r_{0}\right)$ is constant, there is a different wave with the frequency that matches the local Alfvén frequency $\omega_{\mathrm{A}}\left(r_{0}\right)=k_{z} v_{\mathrm{A}}\left(r_{0}\right)$. The wave's profile in $r$ is a Dirac delta function, $\delta\left(r-r_{0}\right)$, and the profile in $z$ is a sin function. The singularity at $r=r_{0}$ can be removed by taking a twisted magnetic field or by accounting for viscosity and/or resistivity.

In this paper we study the torsional Alfvén wave in the framework of Hall MHD. The MHD and Hall MHD models are different because the first assumes the Hall current zero (which means that the ions are magnetized or tied to the magnetic field lines), while the second accounts for its finiteness. Thus, because of the coupling of thermal pressure effects with the cross-field Hall currents at the ion gyroradius length scales, our results also contain the so-called "finite Larmor radius" effects.

The set of Hall-MHD equations is

$\rho \frac{\mathrm{d} \boldsymbol{v}}{\mathrm{d} t}=-\nabla p+\frac{1}{4 \pi}(\nabla \times \boldsymbol{B}) \times \boldsymbol{B}$ 
$\frac{\partial \boldsymbol{B}}{\partial t}=\nabla \times(\boldsymbol{v} \times \boldsymbol{B})-\nabla \times\left[\frac{\boldsymbol{j}}{n e} \times \boldsymbol{B}\right] ;$

$\frac{\partial \rho}{\partial t}+\nabla \cdot(\rho \boldsymbol{v})=0$

where $\boldsymbol{v}$ is the plasma velocity, $p$ the plasma pressure, $\rho \simeq n m_{i}$ the plasma density, $n$ the number density, $\boldsymbol{B}$ the magnetic field and $\boldsymbol{j}$ the current. We complement this set by the polytropic equation of plasma state

$p \rho^{-\gamma}=$ const.

The waves in the tube can be excited by the perturbations of the tube end driven by random motions in the photosphere or by magnetic reconnection. Before the waves are excited, the system is assumed to be in quasi-static equilibrium, and the total pressure is set to be constant across the thread. Putting $\boldsymbol{v}_{0}=0$ in (1) and assuming that the plasma is isothermal, we obtain the equation that describes the equilibrium:

$\frac{1}{4 \pi}\left(\nabla \times \boldsymbol{B}_{0}\right) \times \boldsymbol{B}_{0}=0$,

where the subscript 0 refers to the equilibrium state. Equation (5) implies that the equilibrium currents $\left(\sim \nabla \times \boldsymbol{B}_{0}\right)$ flow along the magnetic field lines. In the $r$ direction, Eq. (5) is

$\frac{\partial}{\partial r}\left(\frac{B_{0 \varphi}^{2}+B_{0 z}^{2}}{4 \pi}\right)+\frac{B_{0 \varphi}^{2}}{8 \pi r}=0$,

where $B_{0 \varphi}$ is the azimuthal component and $B_{0 z}$ the $z$ component of the background field, $\boldsymbol{B}_{0}$. Equation (6) shows that the gas pressure is constant, but the magnetic pressure is variable inside the tube. The gradient of magnetic pressure is balanced by the tension force that appears due to the twist of magnetic field lines. We have to account for Eq. (6) when we choose a specific magnetic field profile.

We assume that the waves produce small perturbations of the equilibrium, which allows us to write each physical quantity (except for the plasma velocity that is set to zero in the equilibrium) as a sum of a background quantity (denoted with the subscript $0)$ and a small perturbation:

$\rho=\rho_{0}+\rho(\boldsymbol{r}, t)$

$p=p_{0}+p(\boldsymbol{r}, t)$

$\boldsymbol{B}=\boldsymbol{B}_{0}(\boldsymbol{r})+\boldsymbol{b}(\boldsymbol{r}, t)$

$\boldsymbol{v}=\boldsymbol{v}(\boldsymbol{r}, t)$

$n=n_{0}+n(\boldsymbol{r}, t)$

$$
\boldsymbol{j}=\boldsymbol{j}_{0}(\boldsymbol{r})+\boldsymbol{j}(\boldsymbol{r}, t)
$$

To find the governing equation for torsional Alfvén waves, we introduce (7)-(9) into (1)-(3), retaining both background quantities and perturbations. Because the perturbations are small, we apply a standard procedure of linearization, neglecting nonlinear terms (products of perturbations):

$\rho_{0} \frac{\partial \boldsymbol{v}}{\partial t}=-\nabla p+\frac{1}{4 \pi}(\nabla \times \boldsymbol{b}) \times \boldsymbol{B}_{0}+\frac{1}{4 \pi}\left(\nabla \times \boldsymbol{B}_{0}\right) \times \boldsymbol{b} ;$

$\frac{\partial \boldsymbol{b}}{\partial t}=\nabla \times\left(\boldsymbol{v} \times \boldsymbol{B}_{0}\right)-\nabla \times\left[\frac{\boldsymbol{j}_{0}}{n_{0} e} \times \boldsymbol{b}+\frac{\boldsymbol{j}}{n_{0} e} \times \boldsymbol{B}_{0}\right] ;$

$\frac{\partial \rho}{\partial t}+\nabla \cdot\left(\rho_{0} \boldsymbol{v}\right)=0$ where the quantities with subscript 0 are background quantities and the rest are perturbations. Then we linearize the derivative of (4) with respect to time, which gives

$\frac{\partial p}{\partial t} \rho_{0}^{-\gamma}-\gamma p_{0} \rho_{0}^{-\gamma-1} \frac{\partial \rho}{\partial t}=0$

Eliminating $\partial \rho / \partial t$ here by the use of (12), we obtain

$\frac{\partial p}{\partial t}=-\gamma p_{0} \nabla \cdot \boldsymbol{v}$

As the plasma is homogeneous in the $z$ direction, the perturbations can be Fourier-analysed in $z$. Since we are interested in the torsional waves with the azimuthal wave number $m=0$, the wave quantities have the following form:

$f(r, z, t)=f(r) \mathrm{e}^{\mathrm{i}(k z-\omega t)}$,

where $\omega$ is the frequency of the wave, $k$ the wavenumber in the $z$ direction, and $f(r)$ the radial wave profile.

Before going further, we have to clarify the notions that we use. The notion of magnetic shear wave defines a wave in which the wave magnetic fields are normal to the local gradients of the wave profile, such that there are no compressive magnetic and velocity perturbations but only shear ones. In ideal MHDs, the torsional Alfvén wave has only $b_{\varphi}$ and $v_{\varphi}$ components. The torsional wave with $m=0$ is also a shear wave, because in any point $(z, r, \varphi)$ the wave quantities $b_{\varphi}$ and $v_{\varphi}$ are perpendicular to the local gradient of the wave profile, which lies in the $z-r$ plane.

In the Hall MHD, the $m=0$ "torsional" Alfvén wave with short cross-field wavelengths is strictly speaking no longer a torsional or shear wave, because it also has the radial $\left(v_{r}\right.$ and $\left.b_{r}\right)$ and the axial $\left(b_{z}\right.$ and $\left.v_{z}\right)$ components, coupled to the main shear/torsional $b_{\varphi}$ and $v_{\varphi}$ components. Nevertheless, since the minor components $v_{r}, b_{r}, b_{z}$, and $v_{z}$ are much smaller than the main ones, we call this mode torsional.

Some simplifying assumptions have to be considered to make the problem analytically tractable. The ones we use are to neglect $b_{r}$ and $v_{z}$. Indeed, putting $b_{r}$ to zero can be justified by the equation $\nabla \cdot \boldsymbol{b}=0$, which can be written as

$\frac{1}{r} \frac{\partial}{\partial r}\left(r b_{r}\right)+\mathrm{i} k b_{z}=0$,

implying that $b_{r} / b_{z} \simeq \lambda_{\perp} / \lambda_{\|}$, where $\lambda_{\|}$is the wavelength along the tube's axis and $\lambda_{\perp}$ is the wavelength perpendicular to it. It is important to notice that $\lambda_{\perp}$ is of the same order as $R$ (tens of meters), while $\lambda_{\|}$is about $10^{6} \mathrm{~m}$. Thus, $\lambda_{\perp} / \lambda_{\|} \ll 1$, which implies that $b_{r} / b_{z} \ll 1$, so $b_{r}$ can be ignored. Here $v_{z}$ is omitted for the same reasons, namely a large mismatch between the parallel and cross-field length scales in the torsional Alfvén wave we consider, although the algebraic justification is not so straightforward.

With these approximations, and using (14) to eliminate $p$, we write the $r$ and $\varphi$ components of Eqs. (10) and (11) as

$$
\begin{aligned}
-\rho_{0} \omega^{2} v_{r}= & \frac{\partial}{\partial r}\left(\frac{\gamma p_{0}}{r} \frac{\partial}{\partial r}\left(r v_{r}\right)\right) \\
& +\frac{\mathrm{i} \omega}{4 \pi}\left[\frac{\partial}{\partial r}\left(B_{0 z} b_{z}\right)+\frac{1}{r^{2}} \frac{\partial}{\partial r}\left(r^{2} B_{0 \varphi} b_{\varphi}\right)\right] \\
-\rho_{0} \omega^{2} v_{\varphi}= & \frac{\omega k}{4 \pi} B_{0 z} b_{\varphi} \\
0=\mathrm{i} k B_{0 z} v_{r}- & \frac{k^{2} c B_{0 z}}{4 \pi n_{0} e} b_{\varphi}
\end{aligned}
$$




$$
\begin{aligned}
-\mathrm{i} \omega b_{\varphi}= & -\frac{\partial}{\partial r}\left(B_{0 \varphi} v_{r}\right)+\mathrm{i} k B_{z} v_{\varphi} \\
& +\frac{\mathrm{i} k c}{4 \pi n_{0} e}\left[\frac{\partial}{\partial r}\left(B_{0 z} b_{z}\right)+\frac{2}{r} B_{0 \varphi} b_{\varphi}\right],
\end{aligned}
$$

where 0 in subscripts indicates background quantities.

Next, we reduce the set of Eqs. (17)-(20) to one differential equation. We multiply (17) by $4 \pi /(\mathrm{i} \omega)$ and $(20)$ by $4 \pi n_{0} e /(\mathrm{i} k c)$, and then subtract eliminating $b_{z}$. The result is

$$
\begin{gathered}
\frac{\partial}{\partial r}\left(B_{0 \varphi} b_{\varphi}\right)-\mathrm{i} \frac{4 \pi}{\omega} \frac{\partial}{\partial r}\left(\frac{\gamma p_{0}}{r} \frac{\partial}{\partial r}\left(r v_{r}\right)\right)-\frac{4 \pi n_{0} e}{c} B_{0 z} v_{\varphi} \\
-\frac{\mathrm{i}}{k} \frac{4 \pi n_{0} e}{c} \frac{\partial}{\partial r}\left(B_{0 \varphi} v_{r}\right)=\mathrm{i} 4 \pi \rho_{0} \omega v_{r}+4 \pi n_{0} e \frac{\omega}{k c} b_{\varphi} .
\end{gathered}
$$

From (18) and (19), we express $v_{\varphi}$ and $b_{\varphi}$ in terms of $v_{r}$ :

$b_{\varphi}=\mathrm{i} \frac{4 \pi n_{0} e}{k c} v_{r}$

$v_{\varphi}=-\frac{\mathrm{i}}{\omega} \frac{e B_{0 z}}{M c} v_{r}$

Using (22) and (23) in (21), we obtain the following equation for $v_{r}$ :

$\rho_{\mathrm{i}}^{2} \frac{\partial}{\partial r}\left(\frac{1}{r} \frac{\partial}{\partial r}\left(r v_{r}\right)\right)=\left(\frac{v_{\mathrm{A}}^{2}}{v_{\mathrm{A} 0}^{2}}-\frac{\omega^{2}}{\omega_{\mathrm{ci}}^{2}}-\frac{\omega^{2}}{k^{2} v_{\mathrm{A} 0}^{2}}\right) v_{r}$.

Here $v_{\mathrm{A}}=B_{0 z}(r) / \sqrt{4 \pi \rho_{0}}$ is the Alfvén speed as a function of $r, v_{\mathrm{A} 0}=B_{0 z}(0) / \sqrt{4 \pi \rho_{0}}$ is the Alfvén speed at $r=0, \omega_{\mathrm{ci}}=$ $e B_{0 z}(0) / M c$ is the ion cyclotron frequency at $r=0, \rho_{\mathrm{i}}=v_{\mathrm{T}} / \omega_{\mathrm{ci}}$ is the ion Larmor radius at $r=0$, and $v_{\mathrm{T}}=\sqrt{\gamma p_{0} / \rho_{0}}$ is the thermal speed. Since the ion cyclotron frequency in the solar corona is of the order of $10^{5} \mathrm{~Hz}$ and the Alfvén frequency in coronal loops is much lower, in the range $0.01-10 \mathrm{~Hz}$, we neglect $\omega^{2} / \omega_{\mathrm{ci}}^{2}$ in the above equation. As a result, we obtain:

$\rho_{\mathrm{i}}^{2} v_{\mathrm{A} 0}^{2} \frac{\partial}{\partial r}\left(\frac{1}{r} \frac{\partial}{\partial r}\left(r v_{r}\right)\right)+\left(\frac{\omega^{2}}{k^{2}}-v_{\mathrm{A}}^{2}\right) v_{r}=0$.

This is the eigenmode equation for torsional Alfvén waves in current threads. It gives the equation for the wave amplitude in $r$ when the Larmor radius $\rho_{\mathrm{i}}$ is taken as a finite quantity. In the limit $\rho_{\mathrm{i}}=0$, when the particles are tied to the magnetic field lines, we obtain

$$
\left(\frac{\omega^{2}}{k^{2}}-v_{\mathrm{A}}^{2}\right) v_{r}=0,
$$

which is the ideal MHD equation for torsional Alfvén waves in inhomogeneous axially symmetric plasma structures. This equation is singular, giving an infinite number of degenerate solutions and a continuous spectrum.

It is useful to write Eq. (25) in a dimensionless form, where all velocities (including $\omega / k$ ) are made dimensionless by the Alfvén velocity $v_{\mathrm{A} 0}$, distances by the ion gyroradius $\rho_{\mathrm{i}}$, and magnetic fields by $B_{0 z}(0)$ - the value of the background magnetic field at $r=0$. Thus we write the governing equation in the dimensionless form as

$\frac{\partial}{\partial r}\left(\frac{1}{r} \frac{\partial}{\partial r}\left(r v_{r}\right)\right)+\left(\frac{\omega^{2}}{k^{2}}-v_{\mathrm{A}}^{2}\right) v_{r}=0$.

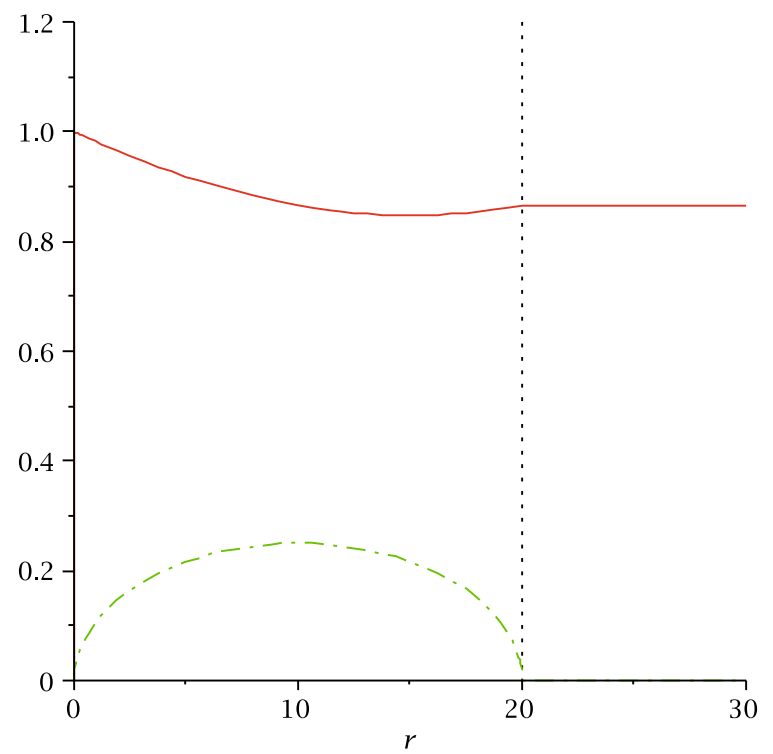

Fig. 2. The plot of $B_{0 z}$ (solid line) and $B_{0 \varphi}$ (dashed-dotted line) fields in the interior and exterior of the current thread for case A. The magnetic field is made dimensionless by the magnetic field at $r=0$, (50 Gauss); $r$ is expressed in units of ion gyroradius at $r=0, \rho_{\mathrm{i}}=27 \mathrm{~cm}$. The expressions for the magnetic field components are given by Eqs. (28) and (29). The dotted line marks the boundary between the interior and the exterior of the tube.

\section{Results}

In this section we present the solutions of the (27) obtained for two particular profiles of the magnetic field. First we specify the loop parameters, used to calculate the dimensional quantities, defined at the end of the previous section. The number density inside and outside the loop is $3 \times 10^{9} \mathrm{~cm}^{-3}$, the temperature is $2 \times 10^{6} \mathrm{~K}$, and the magnetic field at $r=0$ is $B_{0 z}(0)=50$ Gauss. Hence, $v_{\mathrm{A} 0}=2 \times 10^{8} \mathrm{~cm} / \mathrm{s}$ and $\rho_{\mathrm{i}}=27 \mathrm{~cm}$.

A. The first profile for the magnetic field is

$B_{0 \varphi}= \begin{cases}0.025 \sqrt{r(20-r)}, & 0 \leq r \leq 20 \\ 0, & 20 \leq r\end{cases}$

Using this profile for $B_{0 \varphi}, B_{0 z}$ is specified by Eq. (6). In this case, we get

$B_{0 z}= \begin{cases}\sqrt{\frac{r^{2}}{800}-\frac{3 r}{80}+1}, & 0 \leq r \leq 20 ; \\ \sqrt{3} / 2, & 20 \leq r .\end{cases}$

Both components of the magnetic field are shown in Fig. 2. It can be seen that, as $r$ increases, $B_{0 z}$ decreases to a minimum at $r=15$ gyroradii, then goes up again until it reaches a constant plateau. For $r>20$ gyroradii, $B_{0 z}$ is constant. At $r=0 B_{0 \varphi}$ is zero, then it increases to $r=10$ and decreases to zero at $r=20$; for $r>20, B_{0 \varphi}=0$. The maximum value of $B_{0 \varphi}$ reaches $25 \%$ of the value of $B_{0 z}$ at $r=0$, so it is quite significant.

For the profile (28), the piecewise solutions to Eq. (27), which are regular at the origin and zero at infinity, are given by the expressions:

$v_{r}(r)=\left\{\begin{array}{cc}\operatorname{HeunB}\left(2,-\frac{3 \mathrm{i} \sqrt{5}}{2^{1 / 4}}, \frac{115 \sqrt{2}}{4}-20 \sqrt{2}\left(\frac{\omega}{k}\right)^{2}, 0, \frac{\mathrm{i} \sqrt{5}}{2^{1 / 4} 10} r\right) \\ \times r e^{\sqrt{2} r(r-30) / 80}, & 0 \leq r \leq 20 ; \\ c_{1} K_{1}\left(\sqrt{\frac{3}{4}-\left(\frac{\omega}{k}\right)^{2}} r\right), & 20 \leq r,\end{array}\right.$ 


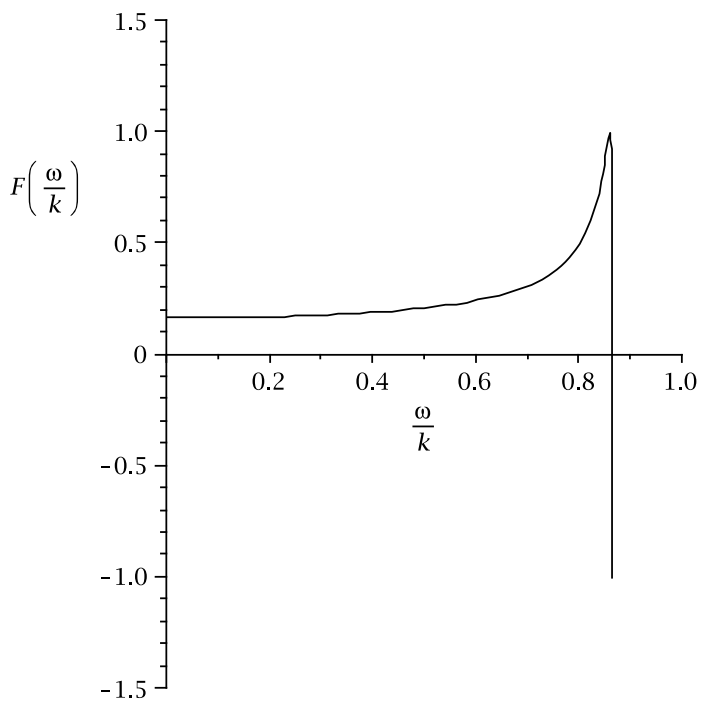

Fig. 3. The dispersion function as a function of the phase velocity, $\omega / k$, for the case A. The phase velocity is expressed in units of $v_{\mathrm{A} 0}=$ $2 \times 10^{8} \mathrm{~cm} / \mathrm{s}$. It is obvious that the dispersion function intersects the horizontal axis in just one point, so the dispersion equation admits only one solution.

where HeunB is a bi-confluent Heun function, $K_{1}$ is the firstorder Kummer function. The phase speed $\omega / k$ and the constant $c_{1}$ are to be determined from the matching conditions at $r=20$, where the solution and its derivatives must be continuous. After the matching conditions are applied, we obtain two algebraic equations with two unknowns, $c_{1}$ and $\omega / k$. Eliminating $c_{1}$ from the two equations, we get the dispersion equation, $F_{1}(\omega / k)=0$, which is a transcendental equation in $\omega / k$. The dispersion function $F_{1}(\omega / k)$ is plotted in Fig. 3. The solutions to the dispersion equation give the possible phase velocities of the trapped modes propagating in the system. The current thread acts like a waveguide and only allows some waves, which are compatible with its geometry. As Fig. 3 shows, for this profile of magnetic field, the dispersion equation only has one solution. We calculated it numerically and obtained $\omega / k=0.865$. This value lies between the minimum of the Alfvén speed inside the tube and the value of the Alfvén speed outside the tube.

The profile for $v_{r}(r)$, corresponding to the mode with $\omega / k \simeq$ 0.865 (which is also the fundamental mode), is plotted in Fig. 4, after $c_{1}$ was calculated from the boundary conditions. It can be seen from Fig. 4 that the amplitude of the mode reaches a maximum in the interior of the cylinder. After that, the wave amplitude starts to decrease, going to zero with growing $r$.

Next, we define the "well-confining condition" and calculate the radius of the cylinder in which the mode is confined well. This is obtained by calculating the safety factor of localization

$q(r)=\frac{\int_{0}^{r} b_{\varphi}^{2} \mathrm{~d} r}{\int_{0}^{\infty} b_{\varphi}^{2} \mathrm{~d} r}$.

We consider the mode to be localized inside a cylinder with radius $r^{*}$ if $q\left(r^{*}\right)=0.9$. For $r=20$, we get $q=0.61$, so the mode is not confined well in the interior of the current thread. The radius of the cylinder that confines the mode is $r^{*} \simeq 33$ gyroradii, which is not much larger than the current thread radius.

We have to mention that the number of trapped modes depends on the magnitude of the azimuthal field. By increasing $B_{0 \varphi}$ (and hence, the amount of twist), we obtain more modes. We can also decrease the azimuthal field, such that we do not obtain

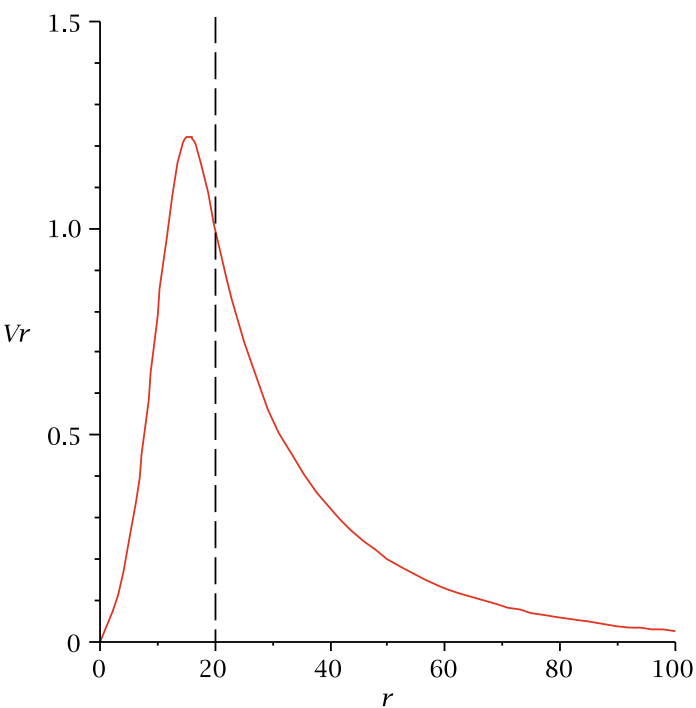

Fig. 4. The amplitude of the radial velocity, $v_{r}(r)$ for case A. Here $v_{r}(r)$ is made dimensionless by the Alfvén velocity, $v_{\mathrm{A} 0}=2 \times 10^{8} \mathrm{~cm} / \mathrm{s}$, and $r$ by the ion gyroradius, $\rho_{\mathrm{i}}=27 \mathrm{~cm}$, at $r=0$. The dashed line shows the boundary between the interior and exterior of the cylinder.

anymore trapped modes in the system. For the chosen profile, the modes disappear if we take 0.023 instead of 0.025 in (28), in front of the $B_{0 \varphi}$ function in the interior on the tube.

B. The second magnetic field profile we analyse is

$B_{0 \varphi}= \begin{cases}0.008 r, & 0 \leq r \leq 20 \\ 0, & 20 \leq r\end{cases}$

The corresponding $B_{0 z}$, calculated from Eq. (6) is

$B_{0 z}= \begin{cases}\sqrt{1-\frac{2}{15625} r^{2}}, & 0 \leq r \leq 20 \\ 1, & 20 \leq r .\end{cases}$

This profile is plotted in Fig. 5. It can be seen that $B_{0 \varphi}$ is zero at $r=0$, then it increases and goes abruptly to zero at $r=20$. At $r=0 B_{0 z}$ is maximum, then it decreases and goes back to the maximum value at $r=20$. This profile is not physical, as it is not continuous at $r=20$, but it can be considered as a good approximation of a realistic profile. Similar to the profile A, it is localized within 20 gyroradii.

The solutions to Eq. (27) for profile B are

$v_{r}(r)= \begin{cases}\frac{\mathrm{i}}{r} M_{\frac{125 i}{2 \sqrt{2}}\left(1-\left(\frac{\omega}{k}\right)^{2}\right), \frac{1}{2}}\left(\frac{\mathrm{i} \sqrt{2}}{125} r^{2}\right), & 0 \leq r \leq 20 ; \\ c_{2} K_{1}\left(\sqrt{1-\left(\frac{\omega}{k}\right)^{2}} r\right), & 20 \leq r,\end{cases}$

where $M$ is the Whittaker function, $K_{1}$ the first-order Kummer function, and $c_{2}$ the constant to be determined from the matching conditions. The solution satisfies the same boundary conditions: it is regular at the origin and zero at infinity. Applying the same matching conditions at $r=20$ (the solution is continuous, with continuous derivatives), we find the dispersion equation for $\omega / k: F_{2}(\omega / k)=0$. The dispersion function $F_{2}(\omega / k)$ is plotted in Fig. 6. It can be seen that it intersects the axis at a single point, which is calculated to be $\omega / k \simeq 0.996$. As in the case $\mathrm{A}$, the system only allows one trapped mode, with the phase speed between the Alfvén speeds at $r=0$ and $r=20$.

The shape of the eigenmode with the phase speed $\omega / k=$ 0.996 is plotted in Fig. 7. It can be seen that it is very similar 


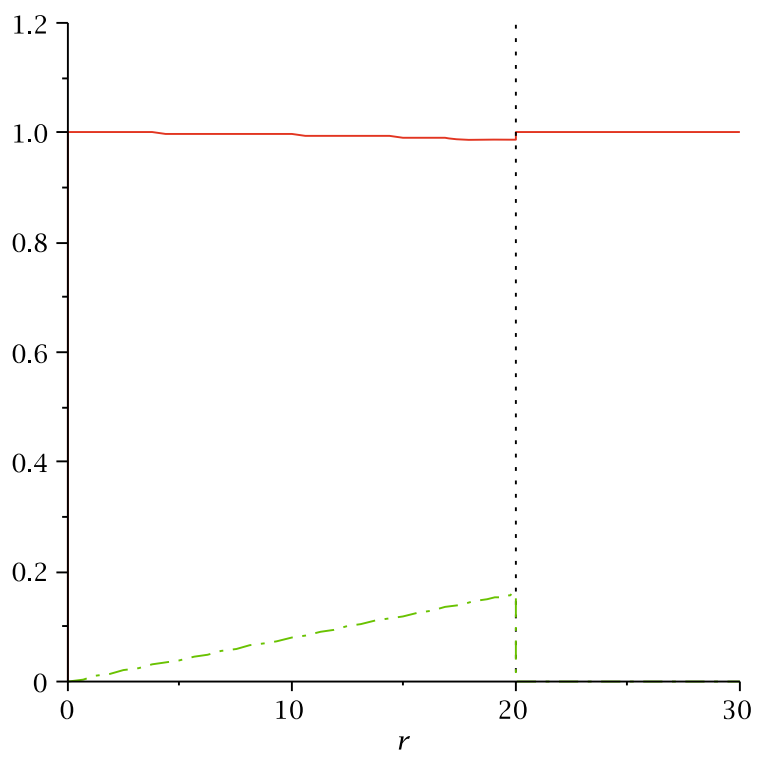

Fig. 5. The plot of $B_{0 z}$ (full line) and $B_{0 \varphi}$ (dashed-dotted line) fields in the interior and exterior of the tube for case B. The magnetic field is made dimensionless by the magnetic field at $r=0,50$ Gauss, and $r$ by the ion gyroradius at $r=0, \rho_{\mathrm{i}}=27 \mathrm{~cm}$. The expressions for the magnetic field components are given by Eqs. (32) and (33). The dotted line marks the boundary between the interior and the exterior of the current thread.

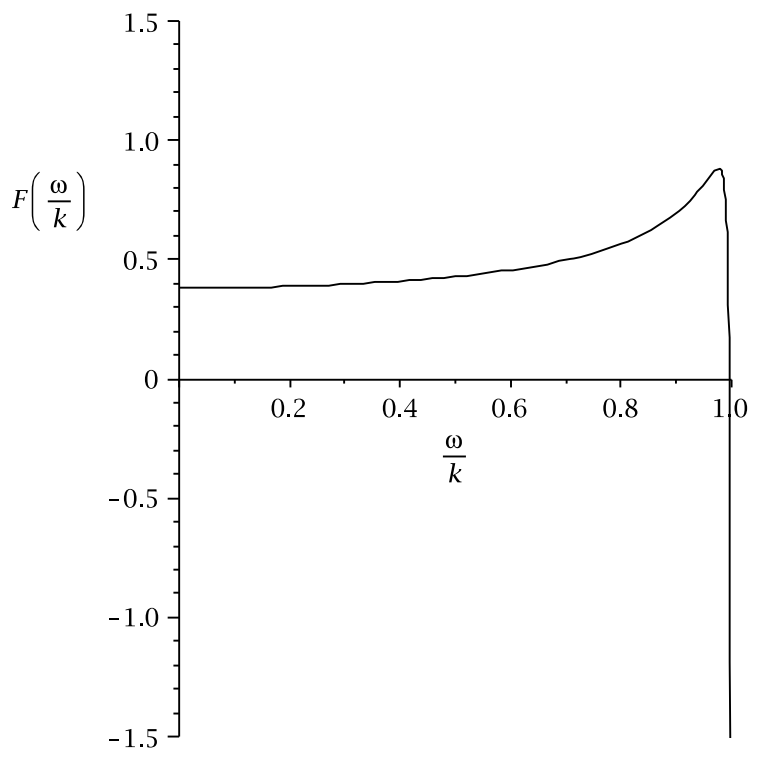

Fig. 6. The dispersion function $F_{2}(\omega / k)$ for case B. The phase velocity $\omega / k$ is expressed in units of $v_{\mathrm{A} 0}=2 \times 10^{8} \mathrm{~cm} / \mathrm{s}$. The dispersion function intersects the horizontal axis in just one point, so the dispersion equation admits only one solution.

to the profile of $v_{r}$ obtained in case A: it has a maximum inside the thread, and then goes to zero as $r$ grows. The difference from case $\mathrm{A}$ is that the amplitude now decreases faster and goes to zero at about 75 gyroradii. This can come from to the more abrupt magnetic field profile. As in case A, it appears that the mode is not well confined in the interior of the cylinder. Using formula 31 we obtain the safety factor $q=0.78$ for $r=20$. The radius of the cylinder that confines the mode is $r^{*}=23.5$, even closer to the current thread radius than in case $\mathrm{A}$.

In case $\mathrm{B}$, the maximum value of $B_{0 \varphi}$ is $16 \%$ of the value of $B_{0 z}$ at $r=0$, so the field does not need to be as twisted as

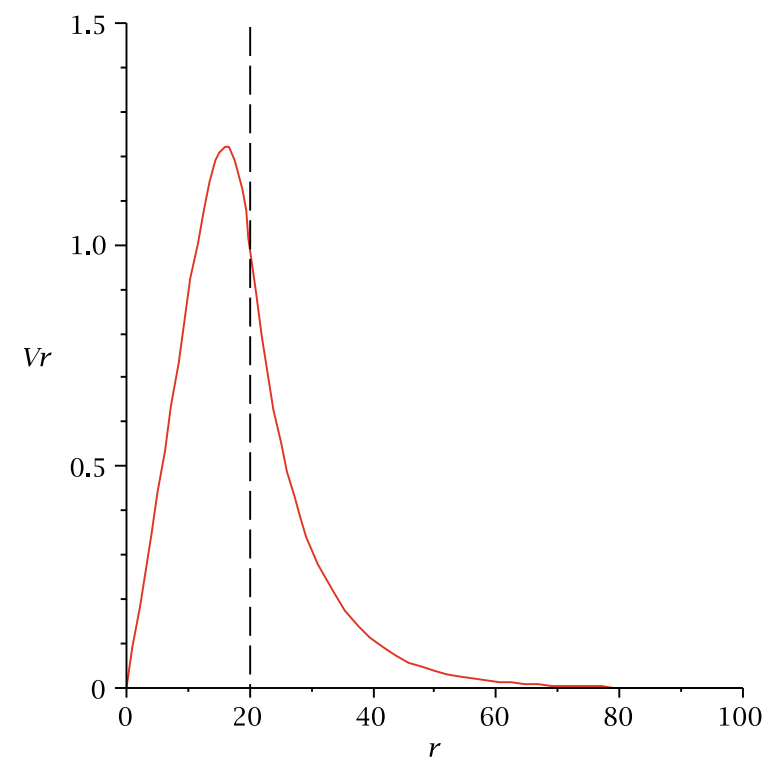

Fig. 7. The amplitude of the radial velocity, $v_{r}(r)$ in $r$, for case B. Here $v_{r}(r)$ is made dimensionless by $v_{\mathrm{A} 0}=2 \times 10^{8} \mathrm{~cm} / \mathrm{s}$ and $r$ by the ion gyroradius, $\rho_{\mathrm{i}}=27, \mathrm{~cm}$ both taken at $r=0$. The dashed line marks the boundary between the interior and exterior of the current thread.

in the previous example. However, this profile is not as realistic as the first one. What seems to be important in obtaining trapped modes is the difference between the minimum value of $B_{0 z}$ inside the tube and the value of $B_{0 z}$ outside, which is reflected in the difference in the Alfvén speed. The difference of $B_{0 z}$ is about the same in the two cases: 0.9 Gauss for case A and 1.3 Gauss for case B.

\section{Conclusions}

In the framework of Hall MHD, we investigated the torsional Alfvén waves in the small-scale current threads in the solar corona. We modelled a thread as a magnetic flux tube with twisted magnetic field lines inside the tube and straight magnetic field lines outside the tube; the density is homogeneous everywhere. The thread's radius is considered to be about 20 gyroradii. We have analysed two particular magnetic field profiles, both localized within $r=20$ gyroradii.

It was shown that the trapped torsional Alfvén waves can propagate in the current thread with sufficient magnetic twist. The waves propagate along the tube's axis $\mathrm{z}$ and have a localized profile across it. The modes are confined in the region of the twisted magnetic field. The wave phase speed is between the minimum value of the Alfvén speed inside the tube and the value of the Alfvén speed outside the tube. Our results show that the number of modes depends on the amount of magnetic twist: the more twisted the tube, the more modes in the system. Also, if the field is not twisted enough the modes can disappear, so there is a threshold twist for their appearance.

The wave profiles we obtained in the present paper are very similar to the wave profiles found in density threads (Copil et al. 2008). In that paper we investigated the trapped torsional Alfvén waves in threads with a homogeneous magnetic field, but inhomogeneous density. The conclusion was that these waves do exist in density threads and are subjected to damping due to viscosity and resistivity. Since the length scales and wave profiles in the current threads are similar to those in the density threads, we suggest that the current thread modes experience a similar 
P. Copil et al.: Torsional Alfvén waves in small scale current threads of the solar corona

damping. It is therefore possible that, as for the density threads, the current threads guiding the torsional Alfvén waves can be places of enhanced coronal heating because of the wave dissipation. However, this has to be analysed quantitatively.

It would also be interesting to study torsional Alfvén waves in threads with both density and magnetic field inhomogeneity to see how these two work together from the point of view of wave trapping and damping.

Acknowledgements. We thank the referee for constructive criticism that helped us to improve the paper. Y.V. acknowledges partial support by STCE (SolarTerrestrial Center of Excellence) under the project "Fundamental science".

\section{References}

Aschwanden M. J., \& Nightingale, R. W. 2005, ApJ, 633, 499

Aschwanden, M. J., Fletcher, L., Schrijver, C. J., \& Alexander, D. 1999, ApJ, 520,880

Aschwanden, M. J., Nakariakov, V. M., \& Melnikov, V. F. 2004, ApJ, 600, 458 Banerjee, D., Teriaca, L., Doyle J. G., \& Wilhelm, K. 1998, A\&A, 339, 208
Copil, P., Voitenko, Y., \& Goossens, M. 2008, A\&A, 478, 921

De Moortel, I., Ireland, J., Walsh R. W., \& Hood, A. 2002, Sol. Phys., 209, 61

Dolla, L., \& Solomon, J 2008, A\&A, 483, 271

Egan, T. F., \& Schneeberger, T. J. 1979, Sol. Phys., 64, 223

Edwin, P. M., \& Roberts, B. 1983, Sol. Phys., 88, 179

Erdélyi, R., \& Carter, B. K. 2006, A\&A, 455, 361

Harrison, R. A., Hood, A. W., \& Pike, C. D. 2002, A\&A, 392, 319

Ishii, T. T., Kurokawa, H., \& Takeuchi, T. T. 1998, ApJ, 499, 898

Leka, K. D., Canfield, R. C., McClymont, A. N., \& Van Driel-Gesztelyi, L. 1996, 462,546

Nakariakov, V. M., Melnikov, V. F., \& Reznikova, V. E. 2003, 412, L7

Nakariakov, V. M., Ofman, L., DeLuca E. E., Roberts, B., \& Davila J. M. 1999, Science, 285,862

Ofman, L. 2009, ApJ, 694, 502

Ofman, L., \& Wang, T. J. 2008, A\&A, 482, L9

Roberts, B., \& Webb, A. R. 1979, Sol. Phys., 64, 77

Spruit, H. C. 1981, A\&A, 102, 129

Spruit, H. C. 1982, Sol. Phys., 75, 3

Vaiana, G. S., Davis, J. M., Giacconi, R., et al. 1973, ApJ, 185, L47

Wang, T. J., \& Solanki, S. K. 2004, A\&A, 421, L33

Wang, T. J., Solanki, S. K., Innes, D. E., Curdt, W., \& Marsch, E. 2003, A\&A, 402, L17

Zaqarashvili, T. V. 2003, A\&A, 399, L15 